\title{
Intermediate-Thrust Arcs and Their Optimality in a Central, Time-Invariant Force Field
}

\author{
A. R. Archenti ${ }^{1}$ and N. X. VINH ${ }^{2}$ \\ Communicated by T. N. Edelbaum
}

\begin{abstract}
This paper presents the general equations of the intermediate-thrust arcs in a general, time-invariant, central force field. Two families of planar arcs, namely, the family of Lawden's spirals in the equatorial plane of an oblate planet and the family of intermediate-thrust arcs in a gravitational field of the form $\mu / r^{n}$, have been considered in detail. The Kelley-Contensou condition has been used to test their optimality condition. It is shown that, in the first case, there exist portions of the arcs at a finite distance satisfying the condition, while, in the second case, the entire family satisfies the condition for $n \geqslant 3$. Hence, in a perturbed Newtonian gravitational force field, the intermediate-thrust arcs, under certain favorable conditions, can be part of an optimal trajectory.
\end{abstract}

\section{Introduction}

The possibility of different gravitational fieids of force existing in space has often been conjectured. Finzi (Ref. 1) has queried the validity of Newton's law at great distances and Tschauner and Fischer (Ref. 2) have investigated the effect of different fields of force on the motion of a particle. These investigation prompted Brookes and Smith (Ref. 3) to examine the problem of optimal rocket trajectories in a general force field. One type of extremal arcs is the family of singular arcs along which the thrust is varying at an intermediate level. This level of thrusting can be low for certain boundary conditions, and hence the arcs of inter-

\footnotetext{
${ }^{1}$ Associate Professor, Departamento de Aeronautica, Universidad Nacional de la Plata, La Plata, Argentina.

${ }^{2}$ Professor, Department of Aerospace Engineering, The University of Michigan, Ann Arbor, Michigan.
} 
mediate thrust (IT-ares) are suitable for orbital transfers using varying low-thrust engines if it can be determined that these arcs are in fact optimal. In a numerical study (Ref. 4), Archenti and Marchal have shown that, in a Newtonian force field, the coplanar arcs always contain one or two portions satisfying the additional optimality condition of Kelley (Ref. 5) and Contensou (Ref. 6). For the limiting case of Lawden's spiral, this optimal portion is at infinity; hence, for all practical applications, Lawden's spiral is nonoptimal.

In this paper, we investigate the optimality of Lawden's spiral in the equatorial plane of an oblate planet and also in the case of a central force field of the type $\mu / r^{n}$. It will be shown that, in the first case, there exists a portion of the spiral which satisfies the Kelley-Contensou optimality condition at a finite distance and, in the second case, the condition is satisfied over the whole arc for $n \geqslant 3$.

\section{Singular Arcs in a Central Force Field}

2.1. Statement of the Problem. A rocket $M$, considered as a mass point, is moving in a central force field with center of attraction at 0 . At a time $t$, the state of the rocket is defined by the position vector $\mathbf{r}(t)$, the velocity vector $\mathbf{v}(t)$, and the characteristic velocity $C$, which gives a measure of the fuel expenditure since the initial time. Specifically,

$$
C=\int_{0}^{t}|\Gamma| d t
$$

where $\Gamma$ is the acceleration due to the propulsive thrust $T$, that is

$$
\mathbf{\Gamma}=\mathbf{T}(t) / m(t),
$$

$m$ being the instantaneous mass of the rocket. The control at any instant $t$ is the thrust vector acceleration $\Gamma$, subject to the constraint

$$
0 \leqslant \Gamma \leqslant \Gamma_{\max }
$$

It is proposed to transfer the rocket, by a proper selection of the control $\Gamma(t)$, from the initial condition $\mathbf{r}_{0}, \mathbf{V}_{0}$ at $t_{0}=0$ to the terminal condition $\mathbf{r}_{j}, \mathbf{V}_{f}$ at the time $t=t_{j}$ such that the final characteristic velocity $C_{f}$ is a minimum. The final time $t_{f}$ may be fixed in advance, or may be subject to the condition $t_{f} \leqslant t_{\max }$, or may be completely free. 
2.2. Equations of the Singular Arcs. First, we have the state equations

$$
\begin{aligned}
d \mathbf{r} / d t & =\mathbf{V}, \\
d \mathbf{V} / d t & =\mathbf{\Gamma}-g(r) \mathbf{r} / r,
\end{aligned}
$$

where $g(r)$ is the gravitational force per unit mass, with $g>0$ for an attracting force field. Using the maximum principle, we introduce the adjoint elements $\mathbf{p}_{r}, \mathbf{p}_{v}, p_{c}$ to form the Hamiltonian

$$
H=\mathbf{p}_{r} \cdot \mathbf{V}+\mathbf{p}_{v} \cdot(\boldsymbol{\Gamma}-g \mathbf{r} / r)+p_{o} \Gamma .
$$

To maximize $H, \boldsymbol{\Gamma}$ must be collinear to $\mathbf{p}_{v}$, and

$$
H=\mathbf{p}_{r} \cdot \mathrm{V}-g\left(\mathbf{p}_{v} \cdot \mathbf{r}\right) / r+\left(p_{v}+p_{c}\right) \Gamma .
$$

$H$ is a constant if the function $g$ is time invariant. For the selection of $\Gamma$, the following rules apply:

$$
\begin{aligned}
& \text { if } p_{v}+p_{c}<0, \text { we select } \Gamma=0 \text { (null-thrust arc), } \\
& \text { if } p_{v}+p_{c}>0 \text {, we select } \Gamma=\Gamma_{\max } \text { (maximum-thrust arc), } \\
& \text { if } p_{v}+p_{c}=0 \text {, we select } \Gamma=\text { variable (intermediate-thrust arc). }
\end{aligned}
$$

Hence, the solution to the problem results in finding the time history of the vector $\mathbf{p}_{v}$, called the primer vector. The primer vector along a maximum-thrust arc is not known. Its integration along a null-thrust arc, in a central force field, was presented by Vinh (Ref. 7). The equations of the primer vector along an intermediate-thrust arc, in a central force field, have been derived by Vinh and Marchal (Ref. 8).

Along an intermediate-thrust arc, we take a rotating coordinate system with origin at the point $M$ such that

$$
M X / / \mathbf{r}, \quad M Z / / \mathbf{r} \times \mathbf{v},
$$

$M X Y Z$ being a right-handed orthogonal trihedral. In this system, we have the components

$$
\mathbf{r}=(r, 0,0), \quad \mathrm{V}=(X, Y, 0), \quad \mathbf{p}_{r}=(a, b, c), \quad \mathbf{p}_{v}=(\alpha, \beta, \gamma) .
$$

Then, we have the equations for the elements along an intermediatethrust arc (Ref. 8)

$$
\begin{gathered}
\alpha^{2}+\beta^{2}+\gamma^{2}=1, \\
\alpha a+\beta b+\gamma c=0, \\
a^{2}+b^{2}+c^{2}=\left[\alpha^{2} g^{\prime} r+\left(1-\alpha^{2}\right) g\right] 1 / r, \\
4 \alpha a r=\left[1-\alpha^{2}-\alpha^{2} g^{\prime \prime} r^{2} /\left(g-g^{\prime} r\right)\right] X+2 \alpha \beta Y,
\end{gathered}
$$


where the prime denotes the derivative taken with respect to $r$. The magnitude of the acceleration control is given by

$$
\begin{aligned}
\Gamma \alpha[3(1 & \left.\left.-\alpha^{2}\right)\left(g-g^{\prime} r\right)-\alpha^{2} g^{\prime \prime} r^{2}\right] \\
= & g\left[\left(g-g^{\prime} r\right)-\left(g^{\prime \prime} r^{2}-5 g^{\prime} r+g\right) \alpha^{2}-4 g^{\prime 2} r^{2} \alpha^{2} / g\right] \\
& -4 a^{2} r\left(g-g^{\prime} r\right)-2 \alpha a\left(g^{\prime \prime} r^{2}+g^{\prime} r-g\right) X+6(\alpha b+\beta a)\left(g-g^{\prime} r\right) Y \\
& +\left(\alpha^{2} / r\right)\left[\left(g^{\prime \prime \prime} r^{3}+2 g^{\prime \prime} r^{2}\right)+g^{\prime \prime 2} r^{4} /\left(g-g^{\prime} r\right)\right] X^{2} \\
& +(2 \alpha \beta / r)\left[g^{\prime \prime} r^{2}-2 g^{\prime} r+2 g\right] X Y \\
& -(1 / r)\left[\left(1+2 \beta^{2}-3 \alpha^{2}\right)\left(g-g^{\prime} r\right)-g^{\prime \prime} r^{2} \alpha^{2}\right] Y^{2} .
\end{aligned}
$$

The problem has the first integrals

$$
\begin{gathered}
a X+b Y-\alpha g=H=\text { const } \\
\mathbf{r} \times \mathbf{p}_{r}+\mathbf{V} \times \mathbf{p}_{v}=\mathbf{k}=\text { const }
\end{gathered}
$$

If the gravitational force is of the form

$$
g(r)=\mu / r^{n}
$$

we have the additional integral of motion

$$
\mathbf{p}_{v} \cdot \mathbf{V}-[2 /(n-1)] \mathbf{p}_{r} \cdot \mathbf{r}+[(n+1) /(n-1)] H t-C=D=\text { const. }
$$

2.3. Optimality Condition. A simple necessary condition for optimality of the intermediate-thrust arc can be obtained by applying the Kelley-Contensou condition (Ref. 5-6)

$$
(-1)^{p}(\partial / \partial \Gamma)\left[\left(d^{2 p} / d t^{2 p}\right)(\partial H / \partial \Gamma)\right] \leqslant 0,
$$

where $p$ is the order of the singularity (in our case, $p=2$ ). Hence, for a general central, time-invariant force field, we have the additional necessary condition for optimality of the intermediate-thrust arc

$$
\left(\alpha / r^{2}\right)\left[3\left(1-\alpha^{2}\right)\left(g-g^{\prime} r\right)-\alpha^{2} g^{\prime \prime} r^{2}\right] \leqslant 0 .
$$

Since $\Gamma \geqslant 0$, we shall use the equivalent condition

$$
P=\left(\alpha \Gamma / r^{2}\right)\left[3\left(1-\alpha^{2}\right)\left(g-g^{\prime} r\right)-\alpha^{2} g^{\prime \prime} r^{2}\right] \leqslant 0 .
$$


2.4. Planar Arcs. In this case, $c=0, \gamma=0$, and we have the equations

$$
\begin{gathered}
\alpha^{2}+\beta^{2}=1, \quad \epsilon= \pm 1, \\
a=(\epsilon \beta / k)\left[H+\left(g-g^{\prime} r\right) \alpha^{3}\right], \\
b=-(\epsilon \alpha / k)\left[H+\left(g-g^{\prime} r\right) \alpha^{3}\right], \\
X=\frac{2 \epsilon \beta\left(g-g^{\prime} r\right)\left\{k^{2}+3 \alpha r\left[H+\left(g-g^{\prime} r\right) \alpha^{3}\right]\right\}}{k\left[3\left(g-g^{\prime} r\right) \beta^{2}-g^{\prime \prime} r^{2} \alpha^{2}\right]}, \\
Y=\frac{\left\{\epsilon \left\{\left(g-g^{\prime} r\right) \beta^{2}\left[-k^{2}+3 \alpha r\left[H+\left(g-g^{\prime} r\right) \alpha^{3}\right]\right]\right.\right.}{k \alpha\left[3\left(g-g^{\prime} r\right) \beta^{2}-g^{2} r^{2} \alpha^{2}\right]}
\end{gathered}
$$

where $r$ is obtained as a function of $\alpha$ from the relation

$$
r\left[H+\left(g-g^{\prime} r\right) \alpha^{3}\right]^{2}=k^{2}\left[g-\left(g-g^{\prime} r\right) \alpha^{2}\right] .
$$

\section{Optimality of Lawden's Spiral in the Equatorial Plane of an Oblate Planet}

Archenti and Marchal (Ref. 4) have shown that the optimal portion of Lawden's spiral, in the case of an inverse-square force field, is at infinity. We propose to show that, in the equatorial plane of an oblate planet, there exist spirals with optimal portion at a finite distance from the center of force. form

In the equatorial plane of an oblate planet, the function $g(r)$ has the

$$
g(r)=\left(\mu / r^{2}\right)\left[1+\epsilon(R / r)^{2}\right]+O\left(\epsilon^{2}\right),
$$

where $R$ is the equatorial radius of the planet; for the Earth, according to Jeffreys (Ref. 9), $\epsilon=1.638 \times 10^{-3}$. For Lawden's spiral, $H \equiv 0$, and Eq. (19) becomes

$$
r\left(g-g^{\prime} r\right)^{2} \alpha^{6}=k^{2}\left[g-\left(g-g^{\prime} r\right) \alpha^{2}\right] .
$$

We define a nondimensional radial distance $\rho$ and a nondimensional parameter $k_{1}$ such that

$$
\rho=r / R, \quad k_{1}^{2}=k^{2} R / \mu .
$$

Hence,

$$
k_{1}{ }^{2} \rho=9 \alpha^{2} /\left(1-3 \alpha^{2}\right)+\epsilon k_{1}^{4}\left(7-15 \alpha^{2}\right) / 27 \alpha^{6}+O\left(\epsilon^{2}\right) .
$$


With this expression for $\rho$, we can write the optimality condition (17) as

$$
\begin{aligned}
Q= & \alpha^{8}\left(9-25 \alpha^{2}+20 \alpha^{4}\right) \\
& +\left(\epsilon k_{1}{ }^{4} / 243 \alpha^{6}\right)\left(80 / 3-124 \alpha^{2}-266 \alpha^{4}+2200 \alpha^{6}-3890 \alpha^{8}+2520 \alpha^{10}\right) \\
& +O\left(\epsilon^{2}\right) \leqslant 0,
\end{aligned}
$$

where

$$
Q=\frac{P \rho}{27}\left[\frac{\rho^{3} R^{3} k_{1}}{\mu} \cdot \frac{3 \beta^{2}\left(3 \rho^{2}+5 \epsilon\right)-2 \alpha^{2}\left(3 \rho^{2}+10 \epsilon\right)}{\left(3 \rho^{2}+5 \epsilon\right)}\right]^{2} .
$$

For real arcs, the value of $\alpha^{2}$ is constrained by

$$
0 \leqslant \alpha^{2} \leqslant \alpha_{s}^{2}, \quad \alpha_{s}{ }^{2}=\left(\rho^{2}+\epsilon\right) /\left(3 \rho^{2}+5 \epsilon\right) .
$$

Hence, the values of $\alpha^{2}$ which limit the range of optimality for Lawden's spirals in the oblate planet case are the roots of the equation

$$
Q\left(\alpha^{2}\right)=0
$$

containing the interval (25).

Consider the space $k_{1}^{4}, \epsilon>0$ (Fig. 1). We propose to find in this space the region in which it is possible to obtain the values of $\rho$ and $\alpha$ from Eqs. (22) and (26) such that these values satisfy Ineqs. (25). This region is then the region where Lawden's arcs can be optimal. Assume that the problem has been solved, and consider a point $\left(k_{10}^{4}, \epsilon_{0}\right)$ in this

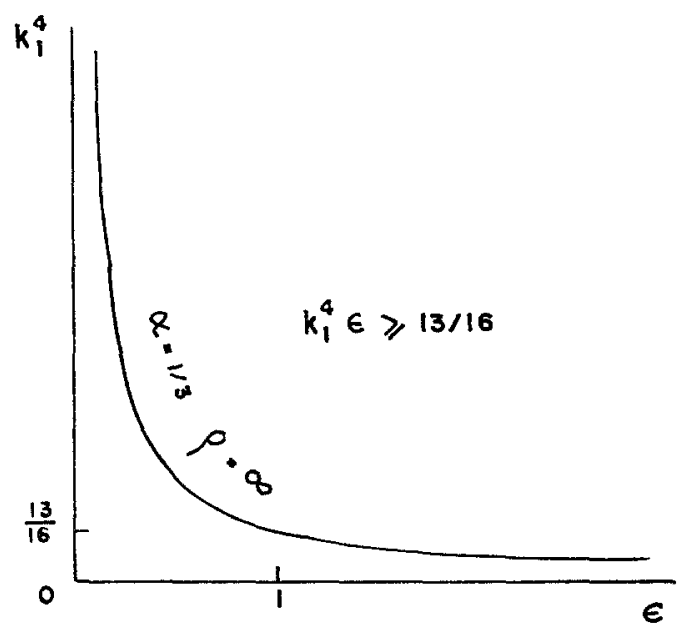

Fig. 1. Region of existence of optimal arcs. 
region. If we move the point along the hyperbola $k_{1}{ }^{4} \epsilon=k_{10}^{4} \epsilon_{0}$, the solution of (26) remains always $\alpha_{0}^{2}$, and hence by (22) we have the same value for $k_{1}^{2} \rho$. Now, if we write the second equation (25) as

$$
\alpha_{s}{ }^{2}=\left[\left(k_{1}{ }^{2} \rho\right)^{2}+k_{1}{ }^{4} \epsilon\right] /\left[3\left(k_{1}{ }^{2} \rho\right)^{2}+5 k_{1}{ }^{4} \epsilon\right],
$$

we see that the interval for $\alpha^{2}$ remains the same. Therefore, if a point $\left(k_{10}^{4}, \epsilon_{0}\right)$ is in the region of optimality, the entire hyperbola $k_{1}{ }^{4} \epsilon=k_{10}^{4} \epsilon_{0}$ passing through this point is contained in this region. Hence, this region is bounded by the hyperbola with the smallest positive value of $\epsilon k_{1}{ }^{4}$ satisfying the optimality condition. From Eq. (26), we have

$$
\epsilon k_{1}^{4}=-P_{9}\left(\alpha^{2}\right) / P_{5}\left(\alpha^{2}\right)>0,
$$

where

$$
\begin{aligned}
& P_{9}\left(\alpha^{2}\right)=243 \alpha^{14}\left(9-25 \alpha^{2}+20 \alpha^{4}\right), \\
& P_{5}\left(\alpha^{2}\right)=\frac{80}{3}-124 \alpha^{2}-266 \alpha^{4}+2200 \alpha^{6}-3890 \alpha^{8}+2520 \alpha^{10} .
\end{aligned}
$$

The polynomial $P_{9}\left(\alpha^{2}\right)$ is always positive, while in the interval $0 \leqslant \alpha^{2} \leqslant \frac{1}{3}$ ( $\frac{1}{3}$ being the maximum value of $\left.\alpha_{s}{ }^{2}\right) P_{5}\left(\alpha^{2}\right)$ has a real pole

$$
\alpha_{R}^{2}=0.27160967 \text {. }
$$

The expression (27) has the limiting values $\frac{13}{16}$ and $\infty$ for $\alpha^{2}$ equal to $\frac{1}{3}$ and $\alpha_{R}{ }^{2}$, respectively. Hence, the region of the $\left(k_{1}{ }^{4}, \epsilon\right)$ plane where optimal intermediate-thrust arcs may occur is bounded by the hyperbola $k_{1}{ }^{4} \epsilon=\frac{13}{16}$, and the optimal values of the parameter $\alpha^{2}$ in the polar equation (22) of the spiral are bounded by

$$
\alpha_{R}{ }^{2} \leqslant \alpha^{2} \leqslant \frac{1}{3} .
$$

We see that the effect of the oblateness of the attracting body has moved the optimal portion of Lawden's spiral from infinity to a finite distance which is, of course, still very large for small values of $\epsilon$.

\section{Optimality of the Planar Singular Arcs in a Gravitational Field of the Form $\mu / r^{n}$}

4.1. Equations of the Singular Arcs. From the general equations of planar, singular arcs, by using $g=\mu / r^{n}$, we have

$$
\left[H r^{n} / \mu+(n+1) \alpha^{3}\right]^{2}=\left(k^{2} r^{n-1} / \mu\right)\left[1-(n+1) \alpha^{2}\right],
$$


with the Kelley-Contensou optimality condition

$$
\begin{aligned}
\operatorname{Pr}^{2} / g^{2}= & \left\{(n+1) /\left[3-(n+3) \alpha^{2}\right]^{2}\right\} \\
& \times\left\{\alpha^{2}\left[a_{1} \alpha^{6}+b_{1} \alpha^{4}+c_{1} \alpha^{2}+d_{1}\right]-\left[4(n-1) H r^{n} \alpha / \mu\right]\right. \\
& \times\left[2(n+3) \alpha^{4}-3(n+3) \alpha^{2}+3\right] \\
& \left.-\left(H^{2} r^{2 n} / \mu^{2} \alpha^{2}\right)\left[3+(n-1)(n+3) \alpha^{4}\right]\right\} \leqslant 0,
\end{aligned}
$$

where

$$
\begin{aligned}
& a_{1}=4(n+1)(n+3)(n-5), \\
& b_{1}=-3(n+3)\left(3 n^{2}-4 n-23\right), \\
& c_{1}=12\left(2 n^{2}-n-19\right), \\
& d_{1}=-27(n-3) .
\end{aligned}
$$

We define the nondimensional radial distance $\rho$ and the nondimensional Hamiltonian constant $K$ by the relations

$$
\rho^{n-1}=k^{2} r^{n-1} / \mu(n+1)^{2}, \quad K \rho^{n}=H r^{n} / \mu(n+1),
$$

and rewrite Eqs. (31) and (32) as

$$
\begin{gathered}
{\left[K \rho^{n}+\alpha^{3}\right]^{2}=\rho^{n-1}\left[1-(n+1) \alpha^{2}\right],} \\
P r^{2} / g^{2}=\left\{(n+1) /\left[3-(n+3) \alpha^{2}\right]^{2}\right\}\left\{\alpha^{2}\left[a_{1} \alpha^{6}+b_{1} \alpha^{4}+c_{1} \alpha^{2}+d_{1}\right]\right. \\
-4(n-1)(n+1) K \rho^{n} \alpha\left[2(n+3) \alpha^{4}-3(n+3) \alpha^{2}+3\right] \\
\left.-\left[(n+1)^{2} K^{2} \rho^{2 n} / \alpha^{2}\right]\left[(n-1)(n+3) \alpha^{4}+3\right]\right\} \leqslant 0 .
\end{gathered}
$$

Let $\alpha_{M}$ be the maximum value of $\alpha$, and let $\rho_{M}$ be the corresponding value for $\rho$; by using Eq. (35), we have

$$
K=[(n-1) /(n+1)] \alpha_{M}{ }^{3} / \rho_{M}{ }^{n}
$$

and

$$
\rho_{M}^{n-1}=[2 n /(n+1)]^{2} \alpha_{M}^{6} /\left[1-(n+1) \alpha_{M}^{2}\right] .
$$

In terms of these new parameters, we write the polar equation of the spiral [Eq. (35)] as

$$
\begin{aligned}
& \left\{\rho^{n} / \rho_{M}{ }^{n}+[(n+1) /(n-1)]\left(\alpha^{3} / \alpha_{M}{ }^{3}\right)\right\}^{2} \\
& \quad=[2 n /(n-1)]^{2}\left(\rho^{n-1} / \rho_{M}^{n-1}\right)\left[1-(n+1) \alpha^{2}\right] /\left[1-(n+1) \alpha_{M}{ }^{2}\right]
\end{aligned}
$$


and the optimality condition as

$$
\begin{aligned}
S= & \left(a_{1} \alpha^{6}+b_{1} \alpha^{4}+c_{1} \alpha^{2}+d_{1}\right)-4(n-1)^{2}\left(\alpha_{M}^{3} / \alpha\right)\left(\rho^{n} / \rho_{M}{ }^{n}\right) \\
& \times\left[2(n+3) \alpha^{4}-3(n+3) \alpha^{2}+3\right] \\
& -(n-1)^{2}\left(\alpha_{M}^{6} / \alpha^{4}\right)\left(\rho^{2 n} / \rho_{M}^{2 n}\right)\left[(n-1)(n+3) \alpha^{4}+3\right] \leqslant 0,
\end{aligned}
$$

where

$$
S=\left[\operatorname{Pr}^{2} /(n+1) g^{2} \alpha^{2}\right]\left[3-(n+3) \alpha^{2}\right]^{2} .
$$

4.2. Lawden's Spiral. This arc is obtained when the Hamiltonian constant $K$ is zero. We have

$$
\rho_{M}=\infty, \quad \alpha_{M}=1 / \sqrt{ }(n+1) .
$$

The polar equation of the spiral is

$$
\rho^{n-1}=\alpha^{6} /\left[1-(n+1) \alpha^{2}\right],
$$

and the optimality condition is

$$
S=a_{1} \alpha^{6}+b_{1} \alpha^{4}+c_{1} \alpha^{2}+d_{1} \leqslant 0 .
$$

4.3. Reversible Arc. This arc was found by Marchal for $n=2$ (Ref. 10). For this limiting case,

$$
\alpha_{M}=0, \quad \rho_{M}=0,
$$

and we have the polar equation of the curve

$$
\rho^{n}+\alpha^{3} / K=0 .
$$

We can take $K=1$ by a change of the reference length and have the equation

$$
\rho^{n}=-\alpha^{3} .
$$

The optimality condition becomes

$$
\begin{aligned}
S= & \left(a_{1} \alpha^{6}+b_{1} \alpha^{4}+c_{1} \alpha^{2}+d_{1}\right)+4(n+1)(n-1) \\
& \times \alpha^{2}\left[2(n+3) \alpha^{4}-3(n+3) \alpha^{2}+3\right] \\
& -(n+1)^{3} \alpha^{2}\left[(n-1)(n+3) \alpha^{4}+3\right] \leqslant 0 .
\end{aligned}
$$

4.4. Optimality of the Planar Singular Ares for $n>2$. The test function $-S\left(\alpha^{2}\right)$ for Lawden's spiral [Eq. (44)] and the reversible 
arc [Eq. 47)] has been computed for different values of $n=3, \ldots, 9$, for $\alpha^{2}$ between zero and its maximum value $\alpha_{M}{ }^{2}$. While the arcs are nonoptimal for $n=2$, because the function is negative, for the case $n \geqslant 3$, the function is always positive. Figure 2 presents the result for Lawden's spiral, and Fig. 3 presents the results for the reversible arc. Hence, we can conclude that, for the case $n \geqslant 3$, Lawden's spiral and the reversible arc always satisfy the Kelley-Contensou optimality condition and thus can be part of an optimal trajectory. These cases are limiting cases of the general arc given by Eq. (39). It is possible to show that, for this general case, the test function $-S\left(\alpha^{2}\right)$ is also positive. The function $S$, given by Eq. (40), is a quadratic function in $\left(\rho / \rho_{M}\right)^{n}$. Its discriminant is given by

where

$$
\Delta=4\left(\alpha_{M}{ }^{6} / \alpha^{4}\right)(n+1)^{2}(n-1)^{2} F,
$$

$$
\begin{aligned}
F= & 4(n-1)^{2} \alpha^{2}\left[2(n+3) \alpha^{4}-3(n+3) \alpha^{2}+3\right]^{2} \\
& +\left[(n-1)(n+3) \alpha^{4}+3\right]\left(a_{1} \alpha^{6}+b_{1} \alpha^{4}+c_{1} \alpha^{2}+d_{1}\right) .
\end{aligned}
$$

Figure 4 plots the values of $F$ for different values of $n$, for $\alpha^{2}$ between 0 and $\alpha_{M}{ }^{2}$. The function $F$ is always negative for $n>2, \alpha^{2}>0$. Hence, the test function $-S\left(\alpha^{2}\right)$ always keeps the same sign which we have shown to be positive for some particular cases.

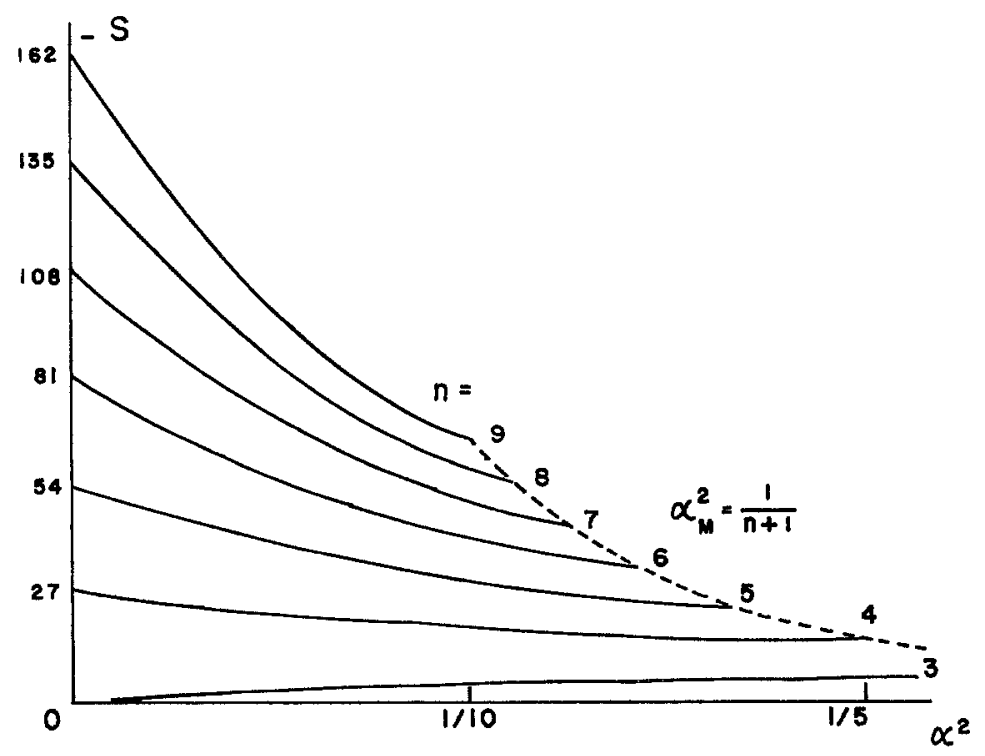

Fig. 2. Plot of the test function $S$ for Lawden's spiral. 


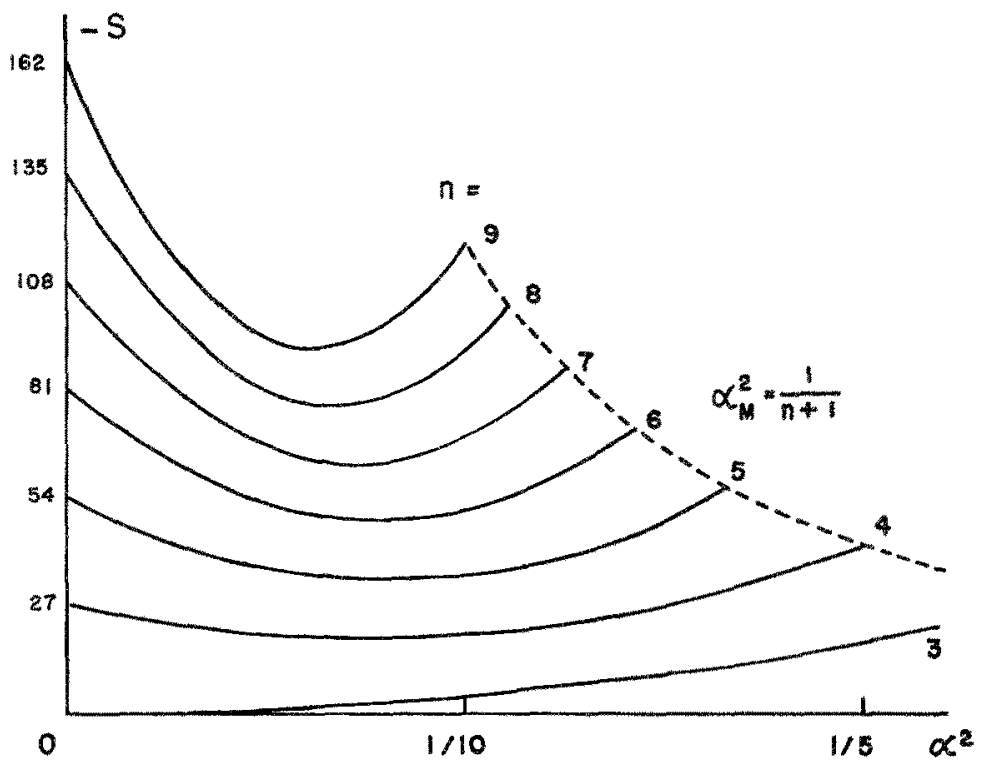

Fig. 3. Plot of the test function $S$ for the reversible arc.

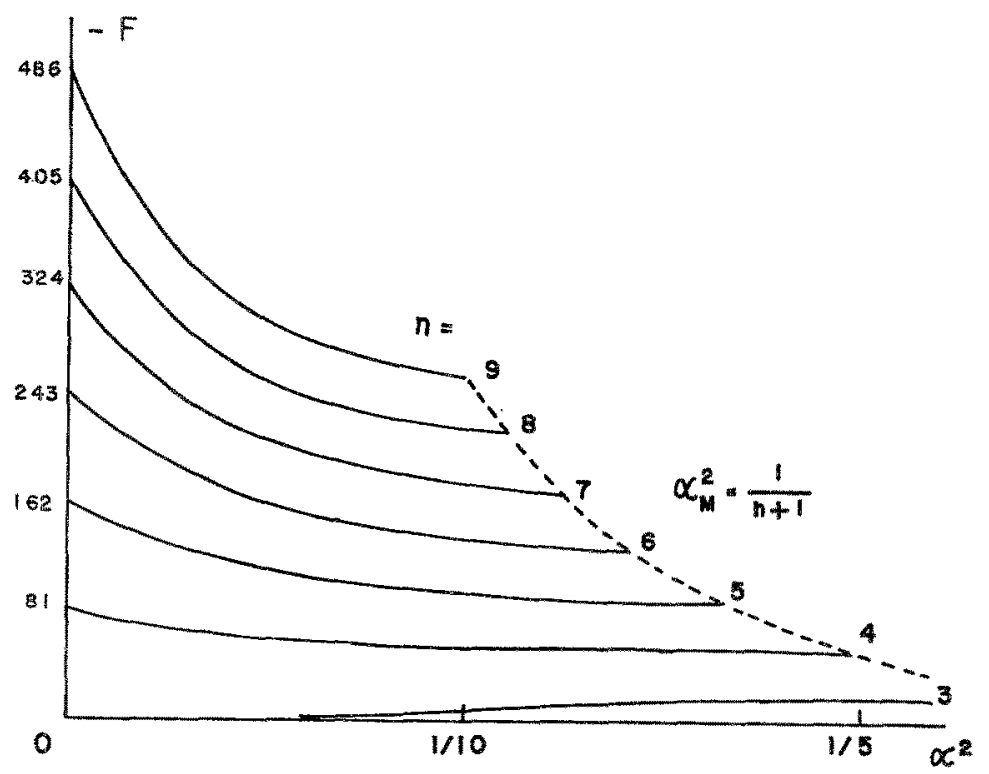

Fig. 4. Plot of the discriminant factor $F$. 


\section{Conclusions}

In this paper, we have derived the general equations of the intermediate-thrust arc (IT-arcs) in a general, time-invariant, central force field. Two families of planar arcs, namely, the family of time free, intermediate-thrust arcs in the equatorial plane of an oblate planet and the family of intermediate-thrust arcs in a gravitational field of the form $\mu / r^{n}$ have been considered. The Kelley-Contensou condition has been used to test their optimality. It is shown that, in the first case, there exist portions of the arcs at a finite distance satisfying the optimality condition, while, in the second case, the entire family satisfies the condition for $n \geqslant 3, \alpha^{2}>0$. Hence, in a perturbed Newtonian gravitational force field, the intermediate-thrust arcs (IT-arcs), under certain favorable terminal conditions, can be part of an optimal trajectory.

\section{References}

1. FinzI, A., On the Validity of Newton's Law at a Long Distance, Monthly Notices of the Royal Astronomical Society, Vol. 127, No. 1, 1963.

2. Tschauner, J., and Fischer, W., Ein Beitrag zur Theorie der Zentralbewegung, Astronautica Acta, Vol. 13, No. 3, 1967.

3. Brookes, C. J., and Sмiтн, J., Optimal Rocket Trajectories in a General Force Field, Astronautica Acta, Vol. 15, No. 3, 1970.

4. Archenti, A., and Marchal, C., Intégration Numérique des Arcs à Poussée Intermédiaire et Étude de leur Optimalité (Dans un Champ Newtonien Central), Paper presented at the 21st Congress of the International Astronautical Federation, Constance, Germany, 1970.

5. Kelley, H. J., Kopp, R. E., and Moyer, H. G., Singular Extremals, Topics in Optimization, Edited by G. Leitmann, Academic Press, New York, 1967.

6. Con'tensou, P., Conditions d'Optimalité pour les Domaines de Maneuvrabilité à Frontière Semi-affine, Paper presented at the Second International Colloquium on Optimization Techniques, Akademgorodok, USSR, 1968.

7. VINH, N. X., Integration of the Primer Vector in a Central Force Field, Journal of Optimization Theory and Applications, Vol. 9, No. 1, 1972.

8. Vinh, N. X., and Marchal, C., The Lawden's Singular Arcs in a Newtonian Force Field, Paper presented at the 21st Congress of the International Astronautical Federation, Constance, Germany, 1970.

9. Jefrreys, H., The Earth, Its Origin, History, and Physical Constitution, Cambridge University Press, Cambridge, 1952.

10. Marchal, C., Généralisation Tridimensionelle et Étude de l'Optimalité des Arcs à Poussée Intermédiaire de Lawden, La Recherche Aérospatiale, Vol. 2, No. 123, 1968. 